Original Research

\title{
Overuse Injury, Substance Use, and Resilience in Collegiate Female Athlete
}

\author{
Chelsea L Martin, PT, DPT ${ }^{1}$ a , Ellen Shanley, PT, PhD, OCS ${ }^{1}$, Chris Harnish, PhD ${ }^{2}$, Amy M Knab, PhD ${ }^{3}$, Shefali \\ Christopher, PT, DPT, LAT, ATC ${ }^{4}$, Srikant Vallabhajosula, PhD ${ }^{4}$, Garrett S Bullock, PT, DPT ${ }^{5}$ \\ ${ }^{1}$ ATI Physical Therapy, ${ }^{2}$ Department of Exercise Science, Mary Baldwin University, ${ }^{3}$ Kinesiology Department, Queens University of Charlotte, 4 \\ Department of Physical Therapy Education, Elon University, ${ }^{5}$ Centre for Sport, Exercise and Osteoarthritis Research Versus Arthritis, University of \\ Oxford; Nuffield Department of Orthopaedics, Rheumatology, and Musculoskeletal Sciences, University of Oxford; Department of Orthopaedic \\ Surgery, Wake Forest School of Medicine \\ Keywords: resilience, overuse injury, substance use, female athlete \\ https://doi.org/10.26603/001c.25760
}

\section{International Journal of Sports Physical Therapy}

Vol. 16, Issue 4, 2021

\section{Background}

Overuse injury is a common stressor experienced by female collegiate athletes and is often underreported. In response, athletes may develop negative coping skills such as substance use. Alternatively, resilience is a modifiable trait that may positively influence response to musculoskeletal injuries and substance use.

\section{Purpose}

To provide an updated epidemiological profile of overuse injury and substance use and examine the relationship between resilience, overuse injury, and substance use among collegiate female athletes.

\section{Design}

Cross-sectional study

\section{Methods}

Two-hundred and thirty female collegiate athletes were classified into overuse injury and resilience groups. Overuse injury, pain, and substance use incidence proportions (IP) were calculated. Kruskal-Wallis analyses were performed to investigate differences in substance use among resilience groups. Analyses of covariance were performed to evaluate differences in overuse injuries, substantial overuse injuries, and time loss injuries, among resilience groups.

\section{Results}

IP for pain was 45.0\% (95\% CI: 38.2-51.9); Overuse injury 52.0\% (45.1-58.9); Alcohol use 35.1\% (28.6-41.6); Electronic cigarette use 19.5\% (14.6-24.9); Cigarette use 2.8\% (6-5.1); and Drug use 3.3\% (0.9-5.8). No significant differences were found between resilience groups for the Oslo Sports Trauma Research Center Overuse Injury Questionnaire (OSTRC) variables (Pain: $p=0.102$; Overuse injury: $p=0.331$; Substantial overuse injury: $p=0.084$; Not playing: $p=0.058)$, alcohol $(p=0.723)$, or combined substance use $(p=0.069)$.

\section{Conclusions}

Pain and overuse injury prevalence is high among female collegiate athletes. Alcohol followed by electronic cigarette use were the most commonly utilized substances. No significant differences were identified in substance use or overuse injury presentation

\footnotetext{
a Corresponding author:

Chelsea L Martin, PT, DPT

ATI Physical Therapy, 200 Patewood Dr C250

Greenville, SC 29615

Email: cleonard12@elon.edu

Phone: (336) 309-5017
} 
between resilience groups, though further investigation is warranted.

\section{Level of Evidence}

\section{INTRODUCTION}

The National Collegiate Athletic Association regulates and organizes sports for 1,200 colleges and universities, and consists of division one (D1), two (D2), and three (D3) programs. ${ }^{1}$ Female participation in collegiate sports has increased in recent decades, with the number of female teams now surpassing men's teams. ${ }^{2}$ Across all divisions, sport participation exposes female collegiate athletes to a substantial amount of stress, in order to meet the demands of being a student-athlete. ${ }^{3,4}$ Stress can be defined as a state of perceived imbalance between demand and available coping mechanisms, and can come from sources such as relationships, academic responsibilities, or physical challenges such as poor performance and injury. When considering physical stressors, overuse injuries are a possible stressor resulting from progressive microtrauma with no specific identifiable event and inadequate recovery. ${ }^{4,5}$ The prolonged onset and longitudinal nature of overuse injuries expose student athletes to additional stress. ${ }^{4,5}$ Recent epidemiological data demonstrates that female collegiate athletes have a higher overuse injury rate compared to male athletes (24.6 versus 13.2 per 10,000 athlete exposures). ${ }^{4}$ In the same study, the authors reported that $50.8 \%$ of overuse injuries did not result in time loss from sport. ${ }^{4}$ Thus, using standard time loss definitions for injury surveillance, along with the higher rate of overuse injuries in females, may contribute to injury underreporting among female collegiate athletes. 6

Due to current injury classification and reporting method limitations, the Oslo Sports Trauma Research Centre Overuse Questionnaire (OSTRC) was developed to capture the spectrum of overuse symptoms and consequence on participation, training, and performance. ${ }^{6}$ Early research has indicated that the OSTRC captures over ten times as many overuse conditions compared to time-loss definitions. ${ }^{6}$ Using the OSTRC, adolescent female gymnastic, basketball, and volleyball athletes reported 8.6 overuse injuries per 1000 athlete exposures ${ }^{7}$; in a separate study using standard injury definitions, adolescent female athletes participating in the same three sports reported substantially lower rates of injury, 1.76-2.74 per 10,000 athlete exposures. ${ }^{8}$ Among female athletes, updated injury epidemiological data that reflects the spectrum of overuse conditions is essential; thus the OSTRC may allow for improved quantification and understanding of overuse injury burden in this population.

In order to manage sport related stressors such as overuse injuries, athletes may use a variety of coping strategies. ${ }^{9}$ Traditional coping strategies employed by collegiate students include academic support, ${ }^{10}$ social support, ${ }^{11}$ leisure activities, ${ }^{12}$ sport and fitness participation ${ }^{13}$ or participation in risk prone activities (i.e. alcohol and other substance use). ${ }^{14,15}$ Injured student-athletes have fewer options to manage their stress due to participation limitations and variable social support. ${ }^{4,16}$ Substance abuse, has been identified as a negative coping strategy. ${ }^{17-19}$ Misuse of substances by athletes including alcohol, cigarettes, and illicit drugs has been documented in previous studies. ${ }^{14,15,20}$ Misuse of substances has been associated with an array of health, social, academic, and performance consequences. ${ }^{17,18}$ Acute health consequences of illicit drug and alcohol use include acute toxic effects, such as overdose, and accidental injury and harm; chronic effects from sustained use include dependence, development of chronic diseases, and increased likelihood of developing mental disorders. ${ }^{21}$ With regard to cigarettes, chronic use has demonstrated associations with cardiovascular disease, chronic obstructive pulmonary disease and various cancers. $^{22}$ During the 1990 s, multiple studies were conducted on alcohol, tobacco, and drug use among college students, but peer-reviewed literature has been sparse in the last 20 years among collegiate athletes. ${ }^{14,15,20}$ Further, a new delivery mechanism for substance use, the electronic cigarette, has shown increased use among college adults. ${ }^{23,24}$ Although electronic cigarettes were originally marketed as a smoking cessation tool, use has increased among nonsmokers despite sparse data on longitudinal health impacts. ${ }^{23}$ Epidemiological substance use data is needed to inform sports medicine, coaching, and support personnel about the negative coping strategies in female collegiate athletes. ${ }^{18}$

In contrast to negative coping skills, positive coping skills may be utilized by athletes. Resilience is a psychological property that has been associated with positive coping skills such as optimism, interconnectedness with social support systems, and higher self-esteem. ${ }^{25}$ Resilience has been conceptualized in various ways, but a common definition is the ability to bounce back despite the presence of stressors. ${ }^{26}$ Resilience is recognized as a personality trait that can change over time, ${ }^{27}$ and may be modified through intervention programs. ${ }^{28}$ Resilience has been observed to have a positive influence on the management of chronic pain, ${ }^{29}$ osteoarthritis, ${ }^{30}$ and following joint replacement surgery. ${ }^{31}$ Further, high levels of resilience are associated with decreased substance use in nonathlete populations. ${ }^{32}$ Nevertheless, research on athlete resilience has not investigated the relationship of resilience on musculoskeletal health or substance use. ${ }^{33}$ Due to stress experienced among collegiate athletes, ${ }^{3,4}$ resilience may be an important, modifiable characteristic. Specifically, resilience potentially can promote positive coping skills for in the presence of an overuse injury or to minimize negative coping skills such as substance use.

Therefore, the purpose of this study was to provide an updated epidemiological profile of overuse injury and substance use and examine the relationship between resilience, overuse injury, and substance use among collegiate female athletes. Additionally, this study examined the relationship between resilience, overuse injury and substance use among this population. 


\section{METHODS}

\section{STUDY DESIGN}

This study was a cross-sectional design that was a subset of a larger study including male and female athletes which sought to investigate collegiate athlete health and well-being. The study included athletes participating in D1, D2 and D3 institutions. The study was approved by the Elon University Institutional Review Board (ID: 20-026).

\section{PARTICIPANTS AND RECRUITMENT}

The questionnaire was administered by the sports medicine staff to current collegiate athletes via email. Study consent was provided by clicking on the email link which provided a copy of the institutional review board consent form prior to completing the questionnaire with assurance of anonymity. The data were managed and de-identified to ensure anonymity using Qualtrics (Qualtrics, Provo, UT), an online survey database. Data collection occurred over eight weeks from August through September 2019. To reduce participant burden, athletes could save responses and complete the questionnaire at any time during the eight week period. Two reminder emails were sent at week two and week six, and coaches gave verbal reminders during week four to increase participation. Inclusion criteria consisted of: 1) enrollment at a participating institution; 2) listed on the official roster of their sport; 3) university email on file with the athletic department. Exclusion criteria consisted of: 1) no consent given to complete the questionnaire; 2 ) club or recreational collegiate athletes; or 3) no longer participating in varsity collegiate athletics.

\section{QUESTIONNAIRE DESIGN}

The questionnaire was designed to capture several aspects of health and well-being among collegiate athletes. The aspects of health and well-being investigated included: 1) athlete general health; 2) lifestyle and academics; 3) pain, injury, and surgery and 4) well-being. ${ }^{34}$ The questionnaire used in this study was adopted from a cricket health and well-being study ${ }^{34,35}$ and was refined following interviews with three collegiate athletes, two collegiate coaches, one collegiate athletic trainer, two collegiate physical therapists, and one sports medicine physician to identify health and well-being issues pertinent to collegiate athletes. The questionnaire was piloted with all the professionals and the athletes were interviewed for final format adaptations. The questionnaire inquired about demographic information including athlete age, height, weight, sports participation, injury history, alcohol and substance use, sleep habits, and health related quality of life. All data were managed and deidentified via Qualtrics software (Qualtrics, Provo, Utah).

\section{MAIN OUTCOME MEASURES}

\section{ALCOHOL, CIGARETTE, ELECTRONIC CIGARETTE AND DRUG USE}

Alcohol use was determined using questions from the Harvard College Alcohol Study (CAS). ${ }^{20}$ Participants were asked the following question: "Have you drank alcohol in the past 30 days?"20 If the participant answered yes, they were asked to fill out a series of questions that included the following: 1) How many occasions have you had alcohol in the past 30 days? Participants could select 1 to 2,3 to 5,6 to 9, 10 to 19 , 20 to 39 or $40+$ occasions as a response. 2) How important is getting drunk as a reason to drink? Answer choices included Very Important, Important, Somewhat Important, Not at all Important. For the remaining questions, participants typed their answers in a text box to respond: 3) How many occasions in the past 30 days did you drink enough to get drunk? 4) How many times have you missed class as a result of drinking? 5) How many occasions have you consumed alcohol in the last 7 days? 6) How many consecutive drinks did you consume during those occasions? ${ }^{20}$ Heavy drinking style was defined as 10 or more drinking occasions in the last 30 days, a motivation to drink to get drunk of somewhat to very important, getting drunk three or more times in the past 30 days, or four or more consecutive drinks in one occasion in the past week. ${ }^{18}$

To determine tobacco use, questions that captured cigarette use from the Harvard CAS were used. ${ }^{15}$ The first question was "Have you ever smoked cigarettes before?"15 If the participant answered yes, they were asked a series of follow up questions that included: 1) How old were you when you first smoked? Answers were typed in a text box. 2) When did you most recently smoke? Answer choices included Never, More than 12 months ago, More than 30 days ago but less than 12 months ago, Less than 30 days ago. 3) Do you smoke regularly? Participants responded yes or no. If the participant answered yes, they were asked 4) How many times a day? Responses were typed in a text box. ${ }^{15}$ Tobacco use questions did not cover smokeless tobacco in this study.

To determine electronic cigarette use, questions determining electronic cigarette use from the Harvard CAS were adapted. ${ }^{15}$ The participants were asked the following question: "Have you ever used e-cigarettes or a vape pen?"15 If the participant answered yes, the following series of questions were asked: 1) How old were you when you first used e-cigarettes or a vape pen? Participants typed their answer in a text box to respond. 2) When did you most recently use e-cigarettes or a vape pen? Answer choices included Never, More than 12 months ago, More than 30 days ago but less than 12 months ago, Less than 30 days ago. 3) Do you use e-cigarettes or a vape pen regularly? Participants responded yes or no. If the participant answered yes, they were asked 4) How many times a day? Responses were typed in a text box. ${ }^{15}$

Drug use was captured using the Harvard CAS drug survey questions ${ }^{20}$ with the following question: Have you used any recreational drugs within the last 30 days? ${ }^{20}$ If the participant answered yes, they were prompted in the next question to indicate frequency: How frequently do you use them? The participants typed their answers in a text box. ${ }^{20}$

\section{THE OSLO SPORTS TRAUMATIC RESEARCH CENTER FOR OVERUSE INJURY QUESTIONNAIRE}

The OSTRC was used to determine the presence of an overuse injury and their effect on sports performance and training. ${ }^{6}$ The OSTRC has been used in a variety of athletic populations, and demonstrates good validity and reliability 
with an internal consistency of $\alpha=0.91 .^{6}$ The questionnaire consists of four questions asking the patient to indicate levels of pain and the impact of pain and injury on sports participation, training volume, and performance. Each question is based on a scale of 0-25 with 0 indicating no overuse injury problem and 25 indicating a severe overuse injury problem. Questions 1 and 4 were scored on a scale of $0-8-17-25$ and questions 2 and 3 were scored on a scale of 0-6-13-19-25. The values utilized indicate a score of 0 representing no problems, whereas a score of 25 represents the maximum level of problems for each question. The intermediate values are scored as such to allow an even distribution from 0-25. The questions were summated for a total score out of 100 .

Participants with and without an overuse injury problem were identified by using total OSTRC score, and categorizing participants into overuse injury (total OSTRC score >0) or no overuse injury (total OSTRC score $=0$ ) groups. Participants with and without pain were identified by calculating question 4 scores only (to what extent have you experienced pain in the last week), and categorizing participants into pain (score $>0$ ) and no pain (score $=0$ ) groups. To identify severe overuse problems, participants were further categorized into substantial overuse injury (score $=25-39$ ) or no substantial overuse injury (score $=>0$ and $<25$ ), and not playing (score $\geqslant 40$ ) or playing (score $<40$ ) groups to differentiate levels of severity of an overuse problem. ${ }^{6}$

\section{RESILIENCE}

Resilience was assessed using the Brief Resilience Scale (BRS). The BRS has previously demonstrated high testretest reliability and validity. ${ }^{26}$ The BRS consists of six questions that are scored from 1 to 5 . Questions are alternated, such that the most resilient response for odd numbered questions is 5 points and for even questions is 1 point. When scored, the even numbered questions are reversed, and all answers are summated for a total possible score of 6 (low resilience) to 30 points (high resilience). Normative data for BRS scores among athletic populations was not available in the peer reviewed literature. Therefore, to determine differences among participants with high or low resilience scores, the mean and standard deviation (SD) of BRS scores were calculated. ${ }^{31}$ Participants who scored 1 SD below the mean were classified as the low resilience group (LR), those with scores within 1 SD of the mean were classified as the normal resilience group (NR), and participants who scored greater than $1 \mathrm{SD}$ above the mean were classified as the high resilience group (HR) for statistical analysis.

\section{STATISTICAL ANALYSES}

Missing data were analyzed through counts, percentages, and visualization through the $\mathrm{R}$ package naniar ( $\mathrm{R}$ Core Team, 2013; R: A language and environment for Statistical Computing, Vienna, Austria. URL http://www.R-project.org/). Missing data was varied (Age $<1 \%$, Resilience $7 \%$, OSTRC $12 \%$, Alcohol last 30 days $9 \%$, Number of alcohol occasions 69\%, Importance of getting drunk 10\%, Occasions of getting drunk 70\%, Combined E-Cig, Cigarette, and Drug use 8-9\%, Frequency of E-Cig, Cigarette, or Drug Use >70\%).
A complete case analysis was performed. However, due to the varied degrees of missing data, only questions with $\leqslant 10 \%$ missing data were statistically analyzed in order to reduce bias. Descriptive data were reported as mean (SD), median (interquartile range), or count (\%). Sport participation was categorized as individual (cross-country, track and field, triathlon, swimming, golf, and dance), field and court (basketball, soccer, tennis, and volleyball), bat and ball (baseball and softball), and collision (football, rugby, and lacrosse $\left.{ }^{36}\right)$. Overuse injury, pain, alcohol, e-cigarette, cigarette and drug incidence proportions (IP) with 95\% confidence intervals $(95 \% \mathrm{CI})$ were calculated using the following formula ${ }^{37}$ :

$$
\begin{aligned}
\text { Variable IP }= & \frac{\text { No of athletes presenting with variable }}{\text { No of all athletes }} \\
& \times 100 \quad \text { ton }
\end{aligned}
$$

Injury and surgery history prevalence was calculated for all female athletes and for each resilience group. A series of Kruskal-Wallis analyses were performed to investigate potential differences between alcohol and amalgamated substance use and resilience groups. An analyses of covariance (ANCOVA) was performed to evaluate potential differences between current overuse injuries, substantial overuse injuries, and time loss injuries, evaluated by the OSTRC, and resilience groups. Confounders controlled for included injury in the prior four weeks and surgery history $(\mathrm{p}<0.05)$. All statistical analyses were performed in $\mathrm{R}$ version 3.5.1.

\section{RESULTS}

One thousand, two hundred and thirty-nine male and female athletes received the survey. Participant recruitment is illustrated in Figure 1. A total of 230 female athletes (38\% response rate) completed the questionnaire and were included in the study (D1: $n=89,39 \%$ response rate; D2: $n$ $=77,33 \%$ response rate; $\mathrm{D} 3: \mathrm{n}=64,28 \%$ response rate). Median age of participants were 19 years (18-20), and the greatest number of participants participated in field and court sports (43.3\%). 51.9\% reported a new injury but no recent surgery (14.8\%). (Table 1)

Pain and overuse injury IP were $45.0 \%$ (95\% CI: 38.2 to 51.9) and 52.0\% (95\% CI: 45.1 - 58.9) respectively. Among all substances, self-reported alcohol use IP was 35.1\% (95\% CI: 28.6 - 41.6); electronic cigarette use IP was 19.5\% (95\% CI: 14.6 - 24.9); cigarette use IP was 2.8\% (95\% CI: 0.6 - 5.1); and drug use IP was 3.3 (95\% CI: 0.9 - 5.8).

BRS scores ranged from 10 to 30 points, with a mean of 21.3 (4.2). $26.2 \%$ of participants $(n=48)$ were classified as LR, 60.7\% $(n=111)$ as NR, and $13.1 \%(n=24)$ as HR. Nearly a third of all athletes reported an overuse injury (HR: $41.7 \%$, LR: 28.7\%), but fewer substantial overuse injuries (HR: 10.5\%, LR: $8.3 \% \%$ ) or inability to play (HR: $8.3 \%$, LR: $10.5 \%$; Table 2). No significant differences were found between resilience groups for any OSTRC variables (Pain: $\mathrm{p}=0.11$; Overuse injury: $\chi^{2}=2.214, \mathrm{p}=0.34$; Substantial overuse injury: $\chi^{2}=4.962, \mathrm{p}=0.09$; Not playing: $\chi^{2}=5.667, \mathrm{p}=0.06$ ).

Among substances, both groups reported similar alcohol use in the last 30 days (HR: 29.1\%, LR: $35.4 \%$ ) and drinking to get drunk as important (HR: $4.2 \%$. LR: $8.3 \%$ ) (Table 2). No significant differences between resilience groups for alcohol 
questions were found (Alcohol last 30 days: $\chi^{2}=0.652, \mathrm{p}=$ 0.73; Drink to get drunk important: $\chi^{2}=5.747, \mathrm{p}=0.06$ ). Electronic cigarette use was more prevalent in LR group (31.3\%) versus HR group (8.3\%), but cigarette and drug use prevalence were similar between groups (Cigarette- HR: 0\%, LR: 2.8\%; Drug- HR: 0\%, LR: 4.2\%; Table 2). No significant difference was found between resilience groups and overall substance use $\left(\chi^{2}=5.356, \mathrm{p}=0.07\right)$.

\section{DISCUSSION}

The findings of this study indicate that pain and overuse injury is a common adverse event that female collegiate athletes experience. Further, alcohol and electronic cigarette use were the most common substances used. While available research indicates that resilience may play a role in decreased substance use ${ }^{38}$ and musculoskeletal health in nonathlete populations, ${ }^{29-31}$ the current study's findings did not demonstrate this relationship.

The results of this study demonstrated that across all sports, 45 out of 100 female athletes reported pain and 52 out of 100 reported an overuse injury, representing a sizable portion of the population. This finding corroborates existing research on incidence proportion among female athletes when using the OSTRC as a measure to capture pain and overuse injuries. ${ }^{39}$ However, the OSTRC has not been widely used among intercollegiate athletes in the United States. When compared to standard methods of injury classification, such as time-loss or need for medical attention, the results of this study suggest that standard injury classification methods are not capturing the spectrum of overuse problems that are impacting athlete performance, overall health and well-being. ${ }^{40}$ Additionally, female collegiate athletes have a higher risk of overuse injuries compared to males, ${ }^{4}$ and differences in pain response have been reported among males and females, ${ }^{41}$ though the exact cause is unknown. These differences in injury presentation and pain response between male and female athletes highlights a need for further exploration among mechanisms of pain and overuse injury in the female athlete population.

Among substances, alcohol was the most prevalent with more than one in three athletes indicating alcohol use in the past 30 days. Comparatively, previous national surveys have asked participants to indicate alcohol use over the past year. ${ }^{18,42}$ Prevalence rates in 1997 and 2001 were $86.0 \%$ and $80.8 \%$ respectively among female athletes ${ }^{18}$; in more recent years among college aged adults, prevalence rates were $70.8 \%$ and $78 \%$ in 2001-2002 and 2012-2013, though female collegiate athlete prevalence was not determined. ${ }^{42}$ The current study's lower prevalence compared to previous research may be due to several factors. First, this study is reflective of a smaller time frame captured; athletes in their competitive seasons are more likely to abstain from drinking, and therefore prevalence may be underestimated. ${ }^{3}$ Additionally, females have consistently demonstrated lower alcohol consumption overall compared to males, though this study did not seek to compare between males and females. ${ }^{42}$ Further, lower consumption may reflect policy changes following the Harvard CAS, including resolutions passed in Congress calling for university presidents to ad-

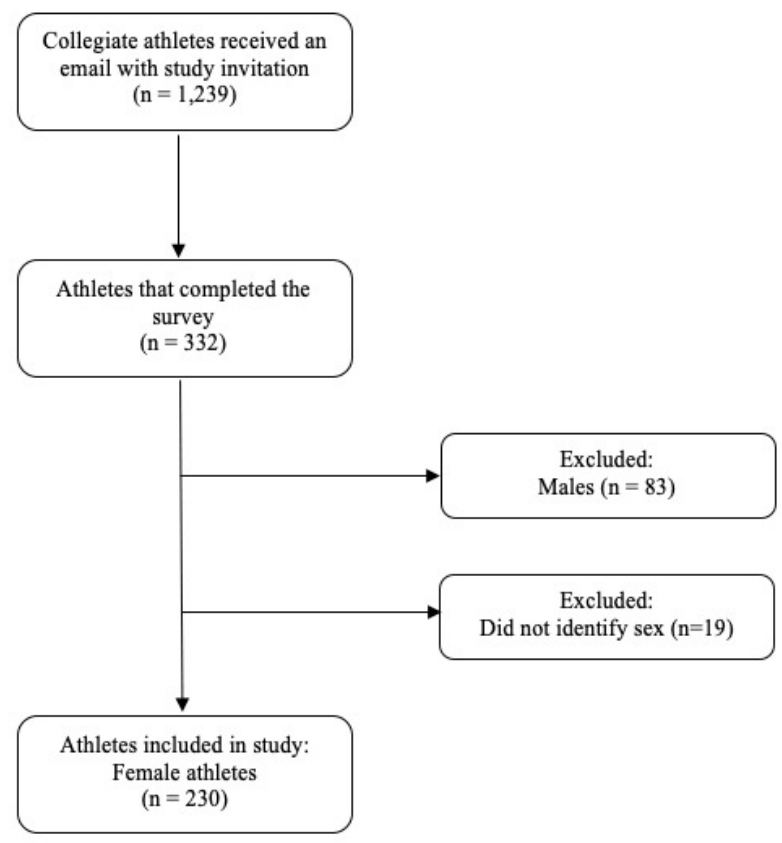

Figure 1. Participation Recruitment Chart

Table 1. Participant Characteristics

\begin{tabular}{|c|c|}
\hline Age (years) & 19(IQR 18-20) \\
\hline Height (m) & 1.7 (SD 0.1) \\
\hline Mass (kg) & $67.6(S D$ 11.9) \\
\hline \multicolumn{2}{|l|}{ Reported Surgery } \\
\hline Yes & 27 (14.8\%) \\
\hline No & 156 (85.3\%) \\
\hline \multicolumn{2}{|l|}{ Reported Injury } \\
\hline Yes & 95 (51.9\%) \\
\hline No & $88(48.1 \%)$ \\
\hline \multicolumn{2}{|l|}{ OSTRC Variables } \\
\hline No Injury & $97(48.0 \%)$ \\
\hline Overuse Injury & $58(28.7 \%)$ \\
\hline Substantial Overuse & $20(9.9 \%)$ \\
\hline Not Playing & $27(13.4 \%)$ \\
\hline \multicolumn{2}{|l|}{ Sport } \\
\hline Individual & $71(31.7 \%)$ \\
\hline Field and Court & $97(43.3 \%)$ \\
\hline Bat, Ball & $40(17.9 \%)$ \\
\hline Collision & $16(7.1 \%)$ \\
\hline \multicolumn{2}{|l|}{ Division } \\
\hline Division 1 & $89(38.7 \%)$ \\
\hline Division 2 & $98(42.6 \%)$ \\
\hline Division 3 & 43 (18.7\%) \\
\hline
\end{tabular}

OSTRC= Oslo Sports Traumatic Research Center Overuse Injury Questionnaire $\mathrm{IQR}=$ interguartile range; $\mathrm{SD}=$ standard deviation; $\mathrm{m}=$ meters; $\mathrm{kg}=$ kilograms

dress heavy alcohol use, and the United States Surgeon 
Table 2. Resilience and Substance Use Profile

\begin{tabular}{|c|c|c|c|c|}
\hline & Overall & Low Resilience & Normal Resilience & High Resilience \\
\hline \multicolumn{5}{|l|}{ OSTRC Variables } \\
\hline \multicolumn{5}{|l|}{ Pain } \\
\hline Yes & $91(45.0 \%)$ & $23(47.9 \%)$ & $44(39.6 \%)$ & $12(50 \%)$ \\
\hline No & $111(55.0 \%)$ & $25(52.1 \%)$ & $67(60.4 \%)$ & $12(50 \%)$ \\
\hline \multicolumn{5}{|l|}{ Overuse Injury Severity } \\
\hline No Injury & $97(48.0 \%)$ & $22(57.9 \%)$ & $59(53.2 \%)$ & $9(37.5 \%)$ \\
\hline Overuse Injury & $58(28.7 \%)$ & $11(28.9 \%)$ & $31(27.9 \%)$ & $10(41.7 \%)$ \\
\hline Substantial Overuse & $20(9.9 \%)$ & $4(10.5 \%)$ & $11(9.9 \%)$ & $2(8.3 \%)$ \\
\hline Not Playing & $27(13.4 \%)$ & $11(28.9 \%)$ & $10(9.0 \%)$ & $3(12.5 \%)$ \\
\hline \multicolumn{5}{|l|}{ Alcohol } \\
\hline Yes & $73(35.1 \%)$ & $17(35.4 \%)$ & $42(37.8 \%)$ & $7(29.1 \%)$ \\
\hline No & $135(64.9 \%)$ & $31(64.6 \%)$ & $69(62.2 \%)$ & $17(70.8 \%)$ \\
\hline \multicolumn{5}{|l|}{ Number of Occasions Last 30 days } \\
\hline 10 or more & $1(1.5 \%)$ & $1(5.9 \%)$ & - & - \\
\hline Less than 10 & $65(98.5 \%)$ & $16(94.1 \%)$ & $42(100 \%)$ & $7(100 \%)$ \\
\hline \multicolumn{5}{|l|}{ Number of Occasions Drunk Last 30 days } \\
\hline 3 or more & $8(13.1 \%)$ & $4(25.0 \%)$ & $4(10.5 \%)$ & - \\
\hline Less than 3 & $53(86.9 \%)$ & $12(75.0 \%)$ & $34(89.5 \%)$ & $7(100 \%)$ \\
\hline \multicolumn{5}{|l|}{ Number of Consecutive Drinks Last 7 days } \\
\hline 4 or more drinks & $22(13.6 \%)$ & $9(19.6 \%)$ & $8(8.3 \%)$ & $5(25.0 \%)$ \\
\hline Less than 4 drinks & $140(86.4 \%)$ & $37(80.4 \%)$ & $88(91.7 \%)$ & $15(75.0 \%)$ \\
\hline \multicolumn{5}{|l|}{ Importance of Getting Drunk } \\
\hline Somewhat Important - Very Important & $30(16.4 \%)$ & $4(8.3 \%)$ & $2(1.8 \%)$ & $1(4.2 \%)$ \\
\hline Not Important at All - Do Not Drink & $176(83.6 \%)$ & $44(91.7 \%)$ & 109 (98.2\%) & $23(95.8 \%)$ \\
\hline \multicolumn{5}{|l|}{ E-Cigarettes } \\
\hline Yes & $41(19.5 \%)$ & $15(31.3 \%)$ & $21(18.9 \%)$ & $2(8.3 \%)$ \\
\hline No & $169(80.5 \%)$ & $33(68.8 \%)$ & $90(81.1 \%)$ & $22(91.7 \%)$ \\
\hline \multicolumn{5}{|l|}{ Smoking } \\
\hline Yes & $6(2.8 \%)$ & $3(6.3 \%)$ & $2(1.8 \%)$ & - \\
\hline No & $205(97.2 \%)$ & $45(93.6 \%)$ & $109(98.2 \%)$ & $24(100 \%)$ \\
\hline \multicolumn{5}{|l|}{ Drugs } \\
\hline Yes & $7(3.3 \%)$ & $2(4.2 \%)$ & $3(2.7 \%)$ & - \\
\hline No & 203 (96.7\%) & 46 (95.6\%) & $108(97.3 \%)$ & 24 (100\%) \\
\hline
\end{tabular}

OSTRC= Oslo Sports Traumatic Research Center Overuse Injury Questionnaire; Data are reported as a count (\%)

Differences in count data between overall and resilience groups are due to missing data

General documented goal of reducing binge drinking by $50 \%$ in $2010 .{ }^{14}$ However, peer-reviewed research on alcohol use among collegiate athletes have been sparse since the Harvard CAS, making it difficult to confirm impact of policy changes and shift in drinking culture among collegiate athletes.

Heavy drinking style is a concern among college athletes. ${ }^{14,18}$ Collegiate athletes are more likely to experience alcohol related harms compared to non-athletes, and are uniquely at risk for sports performance consequences. ${ }^{18}$ In this study, heavy drinking style prevalence as measured independently by frequency, intensity and motivation to get drunk was lower than previous studies of all college students. ${ }^{14}$ However, these findings should be interpreted with caution due to the high percentage of missing data. Previous research has indicated that while college-aged males consume the most alcohol among sex and age groups, females are demonstrating a sharper increase in consumption and heavy episodic drinking, narrowing the gender gap. ${ }^{42}$ This sharp increase among females may be associated with a continued increased rate of females pursuing college level degrees and further economic opportunities, providing a framework for increased exposure and permissive attitudes for heavy drinking. ${ }^{43}$ Peer reviewed research on alcohol 
drinking behaviors among athletes has been sparse in the last 10 years, and substantially more data is needed to expand on our findings to determine accurate prevalence of heavy drinking style among female athletes and related health consequences.

Only $2.8 \%$ of participants reported cigarette use, a finding that demonstrates continued decrease in tobacco use among college athletes and nonathletes since the 1990s. ${ }^{20,44}$ This decrease may be attributed to research, public education, and regulation efforts highlighting the risk of cardiovascular and lung disease as well as perceived negative performance consequences. ${ }^{44,45}$ Additionally, participation in collegiate athletics has demonstrated a protective effect against tobacco use compared to non-athletes. ${ }^{45}$ Drug use was also low at $3.3 \%$ and corroborates previous research indicating that an inverse relationship between participation in sport and drug use exists. ${ }^{44}$ In 2001, marijuana use in the past 30 days among college students was $16.9 \%$ and even lower for all other illicit drugs (7.37\%), much higher than what the current study demonstrates among female athletes. ${ }^{19}$ Our lower prevalence findings may be reflective of athlete awareness on the deleterious effects of drug use on performance, but given a lower response rate on drug related questions $(>70 \%)$, further research is needed to confirm these findings. ${ }^{44}$ Additionally, collegiate athletes are susceptible to random drug testing throughout the year from the NCAA and individual institutions. While studies on impact of drug testing on substance use among collegiate athletes is limited, previous research among high school athletes has indicated that random notification drug testing curtailed substance use short term, and therefore may have similar effects among college athletes. ${ }^{46}$

Interestingly, prevalence of electronic cigarette use was much higher compared to cigarette and drug use; nearly one in five female athletes reported using this delivery mechanism for substances. While data on athlete use is sparse, this represents a much higher prevalence compared to recent use of adults over 18 years old from 2012 to 2013 (1.4-6.8\%). ${ }^{24}$ Electronic cigarette use has not demonstrated the same inverse relationship with sports participation compared to cigarette and drug use. ${ }^{47} \mathrm{~A}$ lack of longitudinal research on adverse health and performance effects, ${ }^{24}$ a shift in marketing strategies geared towards younger consumers, ${ }^{23}$ and fewer regulations in public spaces compared to tobacco products may contribute to perceptions that the product is a safe alternative to cigarettes. ${ }^{39}$ This assumption of safety may reflect the higher prevalence among the current study's cohort and sharp increase in use among adults since the product entered the market in $2006{ }^{24}$ Electronic cigarettes contain toxicants and nicotine ${ }^{24}$ and early studies indicate increased likelihood of future cigarette use to be linked with use of electronic cigarettes. ${ }^{47}$ Additionally, electronic cigarette use has been marketed as a smoking cessation tool, and early evidence indicates that a majority of electronic cigarette users have smoked cigarettes. ${ }^{23}$ Given that the vast majority of the participants in the current study did not report cigarette use, further research is needed to understand motivational factors related to electronic cigarette use in the female collegiate athlete population. Additionally, further research is needed to de- termine the longitudinal health effects of electronic cigarettes.

Little attention has been paid to the impact of psychometric properties such as resilience on musculoskeletal health. While pain and overuse injury represent one of many stressors an athlete faces, ${ }^{39}$ the presence of higher resilience was not associated with differences in pain and overuse injury in this study. Early research has indicated that resilience may have a positive influence on patients with chronic pain, ${ }^{29}$ osteoarthritis ${ }^{30}$ and following joint replacement surgery. ${ }^{31}$ Contrary to these results, this study demonstrated no difference in overuse injuries among different resilience levels in female collegiate athletes. Further research is needed to determine if resilience as a unitary construct is useful to monitor in female athletes, or if additional psychometric properties should be considered to influence overuse injury outcomes. Among substances, differences in use were not determined between resilience levels in the participants. Increased substance use has been cited as a potential negative coping strategy for sport-related pressure and anxiety, including coping with pain, injury, retirement, and performance. ${ }^{18}$ While substance use may be used as a coping strategy among athletes, the lack of association with resilience among the current study findings suggests other explanations may be warranted such as team social dynamics, sorority membership, substance use prior to college, or polydrug use. ${ }^{20}$ However, research on resilience and substance use is still merited given the low percentage of participants that indicated heavy episodic drinking, drug and cigarette use in this study.

This study is not without limitations. The epidemiological profile reported in this study requires further validation to determine the extent of substance use and impact of using the OSTRC to capture overuse injury data among female collegiate athletes. Secondly, this study captured data at one time point. Longitudinal studies may provide a better understanding of substance use, overuse injury, and resilience fluctuations during in-season and off-season periods to improve knowledge of physical and mental stressors and coping strategies athletes experience. Thirdly, given that this was a cross-sectional study there is the potential for recall bias, especially for substance use in the past month, and there is a possibility that athletes may have underreported their substance use. However, use of previously validated outcome measures for overuse injury, substance, and resilience use were used making the results comparable to previous literature. ${ }^{6,15,20}$ The current study response rate was $38 \%$, however this response rate is typical for surveys administered among institutions. ${ }^{48}$ Additionally, given that collision sports were under-represented, and being that the cohort was female only, the results are not generalizable to collision sports or male collegiate athletes. Furthermore, response bias is possible given that athletes interested in study content may be more willing than other athletes to disclose information related to substance or overuse injury. Finally, single method bias is possible given that only an online survey was used to collect data.

\section{CONCLUSION}

In summary, 45 out of 100 female athletes reported pain 
and 52 out of 100 reported an overuse injury, representing a large portion of the population. Regarding recent substance use, alcohol was the most commonly used substance with more than 1 in 3 reporting consumption followed by e-cigarette use at nearly 1 in 5 athletes. The current study findings indicate that resilience did not have any significant associations with pain, overuse injury, or substance use, though further investigation is warranted for associations with heavy episodic drinking and increased data among electronic cigarette, drug, and cigarette use. Notably, given the novelty of electronic cigarettes and high reported use, longitudinal studies on health effects are necessary to provide health education initiatives and information on performance impact. Finally, considering the limitations of current injury definitions to capture injury data, future research should consider use of the OSTRC for further injury surveillance. By improving knowledge on overuse injuries, substance use, and coping strategies, clinicians may be better equipped to provide appropriate interventions and referrals necessary to improve the health and well-being of athletes.

\section{CONFLICT OF INTEREST}

None to disclose

Submitted: February 15, 2021 CDT, Accepted: May 20, 2021 CDT 


\section{REFERENCES}

1. Association NCAA. Our three divisions. http://ww w.ncaa.org/about/resources/media-center/ncaa-101/o ur-three-divisions.

2. NCAA.org. Number of NCAA college athletes reaches all-time high. http://www.ncaa.org/about/res ources/media-center/news/number-ncaa-college-athl etes-reaches-all-time-high. Accessed January 24, 2020 .

3. Martin M. The use of alcohol among NCAA Division I female college basketball, softball, and volleyball athletes. J Athl Train. 1998;33(2):163-167.

4. Yang J, Tibbetts AS, Covassin T, Cheng G, Nayar S, Heiden E. Epidemiology of overuse and acute injuries among competitive collegiate athletes. J Athl Train. 2012;47(2):198-204. doi:10.4085/1062-6050-47.2.198

5. Fuller CW, Ekstrand J, Junge A, et al. Consensus statement on injury definitions and data collection procedures in studies of football (soccer) injuries. Scand J Med Sci Sports. 2006;16(2):83-92. doi:10.1111/ j.1600-0838.2006.00528.x

6. Clarsen B, Myklebust G, Bahr R. Development and validation of a new method for the registration of overuse injuries in sports injury epidemiology: the Oslo Sports Trauma Research Centre (OSTRC) overuse injury questionnaire. Br J Sports Med.

2013;47(8):495-502. doi:10.1136/bjsports-2012-09152 $\underline{4}$

7. Richardson A, Clarsen B, Verhagen EALM, Stubbe $\mathrm{JH}$. High prevalence of self-reported injuries and illnesses in talented female athletes. BMJ Open Sport Exerc Med. 2017;3(1):e000199. doi:10.1136/bmjsem-2 016-000199

8. Schroeder AN, Comstock RD, Collins CL, Everhart J, Flanigan D, Best TM. Epidemiology of overuse injuries among high-school athletes in the United States. J Ped. 2015;166(3):600-606. doi:10.1016/j.jped $\underline{\mathrm{s} .2014 .09 .037}$

9. Crocker PRE, Tamminen KA, Gaudreau P. Coping in sport: a review. Contemporary adv in sport psych. 2015:28-67.

10. Thompson B. How College Freshmen Communicate Student Academic Support: A grounded theory study. Communication Education. 2008;57(1):123-144. doi:10.1080/03634520701576147
11. Brougham RR, Zail CM, Mendoza CM, Miller JR. Stress, Sex differences, and coping strategies among college students. Curr Psychol. 2009;28(2):85-97. doi:1 $\underline{0.1007 / \mathrm{s} 12144-009-9047-0}$

12. Tinsley HEA, Eldredge BD. Psychological benefits of leisure participation: A taxonomy of leisure activities based on their need-gratifying properties. $J$ Counseling Psych. 1995;42(2):123-132. doi:10.1037/00 22-0167.42.2.123

13. Myers SB, Sweeney AC, Popick V, Wesley K, Bordfeld A, Fingerhut R. Self-care practices and perceived stress levels among psychology graduate students. Training and Ed in Professional Psych. 2012;6(1):55-66. doi:10.1037/a0026534

14. Wechsler H, Lee JE, Kuo M, Seibring M, Nelson TF, Lee $\mathrm{H}$. Trends in college binge drinking during a period of increased prevention efforts: findings from 4 Harvard School of Public Health College Alcohol Study Surveys: 1993-2001. J Am College Health. 2002;50(5):203-217. doi:10.1080/07448480209595713

15. Wechsler H, Rigotti NA, Gledhill-Hoyt J, Lee H. Increased levels of cigarette use among college students: a cause for national concern. JAMA. 1998;280(19):1673-1678. doi:10.1001/jama.280.19.16 $\underline{73}$

16. Yang J, Peek-Asa C, Lowe JB, Heiden E, Foster DT. Social support patterns of collegiate athletes before and after injury. J Athl Train. 2010;45(4):372-379. do $\mathrm{i}: 10.4085 / 1062-6050-45.4 .372$

17. Leichliter JS, Meilman PW, Presley CA, Cashin JR. Alcohol use and related consequences among students with varying levels of involvement in college athletics. J Am Coll Health. 1998;46(6):257-262. doi:1 $\underline{0.1080 / 07448489809596001}$

18. Martens MP, Dams-O’Connor K, Beck NC. A systematic review of college student-athlete drinking: Prevalence rates, sport-related factors, and interventions. J Sub Abuse Treatment. 2006;31(3):305-316. doi:10.1016/j.jsat.2006.05.004

19. Mohler-Kuo M, Lee JE, Wechsler H. Trends in marijuana and other illicit drug use among college students: results from 4 Harvard School of Public Health College Alcohol Surveys: 1993-2001. J Am Coll Health. 2003;52(1):17-24. doi:10.1080/0744848030959 $\underline{5719}$ 
20. Wechsler H, Davenport AE, Dowdall GW, Grossman SJ, Zanakos SI. Binge drinking, tobacco and illicit drug use and involvement in collegiate athletics. J Am Coll Health. 1997;45(5):195-200. doi:1 $\underline{0.1080 / 07448481.1997 .9936884}$

21. Degenhardt L, Hall W. Extent of illicit drug use and dependence, and their contribution to the global burden of disease. Lancet. 2012;379(9810):55-70. do $\mathrm{i}: 10.1016 / \mathrm{s} 0140-6736(11) 61138-0$

22. Reardon C, Creado S. Drug abuse in athletes. Sub Abuse and Rehab. August 2014:595-605. doi:10.2147/s ar.s53784

23. Sutfin EL, McCoy TP, Morrell HER, Hoeppner BB, Wolfson M. Electronic cigarette use by college students. Drug and Alcohol Dependence. 2013;131(3):214-221. doi:10.1016/j.drugalcdep.201 3.05 .001

24. Breland A, Soule E, Lopez A, Ramôa C, El-Hellani A, Eissenberg T. Electronic cigarettes: what are they and what do they do? Ann NY Acad Sci. 2016;1394(1):5-30. doi:10.1111/nyas.12977

25. Sarkar M, Fletcher D. Psychological resilience in sport performance: a review of stressors and protective factors. J Sport Sci. 2014;15:1419-1434.

26. Smith BW, Dalen J, Wiggins K, Tooley E, Christopher P, Bernard J. The brief resilience scale: assessing the ability to bounce back. Int J Behav Med. 2008;15(3):194-200. doi:10.1080/10705500802222972

27. Vanhove AJ, Herian MN, Perez ALU, Harms PD, Lester PB. Can resilience be developed at work? A meta-analytic review of resilience-building programme effectiveness. J Occup Organ Psychol. 2016;89(2):278-307. doi:10.1111/joop.12123

28. Zolkoski SM, Bullock LM. Resilience in children and youth: A review. Children and Youth Services Review. 2012;34(12):2295-2303. doi:10.1016/j.childyo uth.2012.08.009

29. Newton-John TRO, Mason C, Hunter M. The role of resilience in adjustment and coping with chronic pain. Rehabilitation Psychology. 2014;59(3):360-365. d oi:10.1037/a0037023

30. Stewart DE, Yuen T. A Systematic Review of Resilience in the Physically Ill. Psychosomatics. 2011;52(3):199-209. doi:10.1016/i.psym.2011.01.036

31. Tokish JM, Kissenberth MJ, Tolan SJ, et al. Resilience correlates with outcomes after total shoulder arthroplasty. J Shoulder and Elbow Surgery. 2017;26(5):752-756. doi:10.1016/i.jse.2016.12.070
32. Acosta MC, Possemato K, Maisto SA, et al. Webdelivered CBT reduces heavy drinking in OEF-OIF veterans in primary care with symptomatic substance use and PTSD. Behavior Therapy. 2017;48(2):262-276. doi:10.1016/j.beth.2016.09.001

33. Galli N, Gonzalez SP. Psychological resilience in sport: A review of the literature and implications for research and practice. International J of Sport and Exercise Psychology. 2015;13(3):243-257.

34. Bullock GS, Collins G, Peirce N, Arden NK, Filbay SR. Physical activity and health-related quality of life in former elite and recreational cricketers from the UK with upper extremity or lower extremity persistent joint pain: a cross-sectional study. BMJ Open. 2019;9(11):e032606. doi:10.1136/bmjopen-201 9-032606

35. Cai H, Bullock GS, Sanchez-Santos MT, et al. Joint pain and osteoarthritis in former recreational and elite cricketers. BMC Musculoskeletal Disorders. 2019;20(1):596.

36. Le RK, Saunders TD, Breedlove KM, et al. Differences in the mechanism of head impacts measured between men's and women's intercollegiate lacrosse athletes. Orthop J Sports Med. 2018;6(11):2325967118807678.

37. Knowles SB, Marshall SW, Guskiewicz KM. Issues in estimating risks and rates in sports injury research. Journal of athletic training. 2006;41(2):207.

38. Morgan JK, Brown J, Bray RM. Resilience as a moderating factor between stress and alcohol-related consequences in the Army National Guard. Addictive Behaviors. 2018;80:22-27. doi:10.1016/j.addbeh.201 $\underline{8.01 .002}$

39. Clarsen B, Bahr R, Heymans MW, et al. The prevalence and impact of overuse injuries in five Norwegian sports: Application of a new surveillance method. Scand J Med Sci Sports. 2015;25(3):323-330. ㅁ oi:10.1111/sms.12223

40. Powell JW, Dompier TP. Analysis of Injury Rates and Treatment Patterns for Time-Loss and NonTime-Loss Injuries Among Collegiate StudentAthletes. J Athl Train. 2004;39(1):56-70.

41. Greenspan JD, Craft RM, LeResche L, et al. Studying sex and gender differences in pain and analgesia: a consensus report. Pain. 2007;132(Supplement 1):S26-S45. doi:10.1016/j.pai n.2007.10.014 
42. Dawson DA, Goldstein RB, Saha TD, Grant BF. Changes in alcohol consumption: United States, 2001-2002 to 2012-2013. Drug Alcohol Depend. 2015;148:56-61. doi:10.1016/j.drugalcdep.2014.12.01 $\underline{6}$

43. Keyes KM, Li G, Hasin DS. Birth cohort effects and gender differences in alcohol epidemiology: a review and synthesis. Alcohol Clin Exp Res.

2011;35(12):2101-2112. doi:10.1111/j.1530-0277.201 1.01562.X

44. Lisha NE, Sussman S. Relationship of high school and college sports participation with alcohol, tobacco, and illicit drug use: A review. Addictive Behaviors. 2010;35(5):399-407. doi:10.1016/j.addbeh.2009.12.03 $\underline{2}$

45. Primack BA, Fertman CI, Rice KR, Adachi-Mejia AM, Fine MJ. Waterpipe and cigarette smoking among college athletes in the United States. J Adolesc Health. 2010;46(1):45-51. doi:10.1016/j.jadohealth.2009.05.0 $\underline{04}$
46. Goldberg L, Elliot DL, MacKinnon DP, et al. Outcomes of a orospective trial of student-athlete drug testing: The student athlete testing using random notification (SATURN) Study. Journal of Adolescent Health. 2007;41(5):421-429. doi:10.1016/j.j adohealth.2007.08.001

47. Bold KW, Kong G, Camenga DR, et al. Trajectories of e-cigarette and conventional cigarette use among youth. Pediatrics. 2018;141(1):e20171832. doi:10.154 2/peds.2017-1832

48. Baruch Y, Holtom BC. Survey response rate levels and trends in organizational research. Human Relations. 2008;61(8):1139-1160. doi:10.1177/001872 $\underline{6708094863}$ 